Original Article

\title{
HESPERIDIN HYDROGEL FORMULATION USING PECTIN-CHITOSAN POLYMER COMBINATION
}

\author{
ANDHI FAHRURR0JI' ${ }^{1}$, DEA THENDRIANI², HAFRIZAL RIZA ${ }^{3}$
}

${ }^{1}$ Departement of Pharmaceutical Technology, Pharmacy Subject, Faculty of Medicine, Tanjungpura University, ${ }^{2}$ Undergraduate Program of Pharmacy Department Tanjungpura University, ${ }^{3}$ Departement of Chemical Pharmacy, Pharmacy Subject, Faculty of Medicine, Tanjungpura University

Email: roji_apt@pharm.untan.ac.id

Received: 09 May 2017 Revised and Accepted: 02 Nov 2017

ABSTRACT

Objective: Hesperidin, a flavonoid glycosides that have been proven to have therapeutic activity to some disease, one of them is colon disease; in addition of its efficacy,low solubility $(<100 \mathrm{mg} / \mathrm{l})$ makes hesperidin slightly absorbed,hence it needs a delivery system which could deliver hesperidin to its therapeutic target. This research aims to obtain an optimum formula for pectin polymer combination which can regulate in vitro hesperidin release.

Methods: Determination of optimum hydrogel formula uses Design Expert 7.0.0 with factorial method design, resulting in pectin-chitosan concentration formula plan-comparison, which are (P3\%: C1\%), (P3\%: C2\%), (P5\%: C1\%), (P5\%: C2\%) respectively. Hydrogel was obtained from a variety of formulas,then evaluation of the entrapment efficiency test, swelling index, in vitro drug release test, mucoadhesive strength were conducted.

Results: Optimum formula with: pectin: chitosan concentration comparison (5: \%: 1\%) have an entrapment efficiency of $96.658 \%$; $\mathrm{k}$ (/hour) swelling index at $\mathrm{pH} 5.0,6.8$, and 7.4, was 34.917, 15.766, and 8.146 respectively; drug release at $\mathrm{pH} 5.0,6.8$, and a medium contained $2 \%$ rat caecum was $0.461,20.116$, and the mucoadhesive strength was $0.184 \mathrm{~N} / \mathrm{cm}^{2}$. Based on the test result using independent $\mathrm{t}$-test sample, actual and prediction value from every test parameter produced by the optimum formula was not significantly different with p-value>0,05.

Conclusion: Combination of pectin-chitosan polymer in hydrogel mucoadhesive regulates hesperidin shesperidin in vitro release, with highest drug release in medium containing $2 \%$ rats caecum which releases $56 \%$ of active substance. Hesperidin hydrogel release mechanism follows Higuchi kinetics. The optimum hesperidin hydrogel formula is the formula with $5 \%$ of pectin and $1 \%$ of chitosan. Based on experimental data value which uses simplex lattice design, optimum hesperidin hydrogel formula has insignificant difference between observed and predicted value ( $\mathrm{p}$ value $>0.05$ )

Keywords: Hesperidin, Hydrogel, Chitosan, Colon, Pectin

(C) 2017 The Authors. Published by Innovare Academic Sciences Pvt Ltd. This is an open access article under the CC BY license (http://creativecommons.org/licenses/by/4.0/) DOI: http://dx.doi.org/10.22159/ijpps.2017v9i12.19816

\section{INTRODUCTION}

Controlled drug delivery system is one of a method to control drug release in order to increase drug effectiveness; and commonly applied to the active substance with low solubility, one of them is hesperidin. it is a flavonoid glycoside which is isolated from citrus plant [1]<smiles>COc1ccc([C@@H]2CC(=O)c3c(O)cc(O[AlH2])cc3O2)cc1O</smiles>

Fig. 1: Hesperidin structure [1]

Hesperidin has been proven to have anti-inflammation antimicrobial, antioxidant, anti-hemorrhoid, and anticancer activity so it could be used in therapy of some colon related diseases such as hemorrhoid, chronic vein insufficiency, colon cancer, and ulcerative colitis [1-6], however, hesperidin has low solubility on digestive tract $(<100 \mathrm{mg} / \mathrm{l})$ as well as low bioavailability $(<25 \%)[1,7]$. Therefore, the correct delivery system is required to increase the bioavailability and therapy effectiveness from hesperidin, one of them is by formulating it into a hydrogel.

Pectin is one of the hydrogel constituent polymer commonly used to deliver drugs to the colon. Previous research showed that ibuprofen release from hydrogel made from pectin decreases with the use of controlled $\mathrm{pH}$ dissolution medium, which indicates that $\mathrm{pH}$ controlled drug release has occurred [8]. Other research showed that hydrogel beads of pectin-zein protects indomethacin from upper gastrointestinal tract conditions and its release was controlled by pectin degradation with pectinase; however, pectin is highly soluble in water, which leads to the development of another polymer with low solubility in water, to produce a strong and effective gel for carrying drug which is entrapped in gastric fluid and small intestine [9-10]. One of the polymers that can be used to overcome this problem is chitosan, which its carboxylic group binds ionically with the chitosan amine group [11]

The study about the role of chitosan as a coating to modify drug release has been conducted to amide pectin hydrogel [12]. Other research which uses pectin polymer in combination with alginate could float on water, $0.1 \mathrm{~N}$ of $\mathrm{HCl}$, and phosphate buffer, which concludes that increase in polymer concentration and crosslink forming time could increase polymerization, but higher pectin concentration lowers drug release up to $12 \mathrm{~h}$, which is caused by the presence of pectinase enzyme [13]. Both drug solubility and drug insolubility in water inside a simulated intestinal condition was significantly reduced compared to pectin hydrogel without chitosan coating. Another research stated that hydrogel, which was formulated from the pectin-chitosan combination is proven to reduce vancomycin release in acidic condition, and increases drug release in a simulatedcolon condition [14]. Based on this research, the author conducts several experiments using hesperidin as an active substance which has low solubility in the digestive tract, but possess several pharmacological benefits which would be formulated into a hydrogel by using pectin-chitosan polymer, in order to maximize its potency in digestive tracts, especially colon. 


\section{MATERIALS AND METHODS}

\section{Materials}

Materials used in this research including hesperidin obtained from Sigma-Aldrich, Batch Number: SLBJ1579V, chitosan (Biotech Surindo, Batch Number: 10A0215. F. HM. CHC), pectin, acetic acid (Merck), sodium acetate (Merck), sodium hydroxide, sodium dihydrogen phosphate, disodium hydrogen phosphate, zinc acetate, and distilled water.

\section{Formulation of hesperidin hydrogel}

Pectin solution was prepared by dissolving pectin in $\mathrm{CO}_{2}$-free distilled water using a magnetic stirrer ( $300 \mathrm{rpm}$ for $15 \mathrm{~min}$ ) at room temperature $\left(25{ }^{\circ} \mathrm{C}\right)$. Chitosan solution was prepared by mixing chitosan with $2 \%$ acetic acid $(\mathrm{b} / \mathrm{v})$, then stirred at $300 \mathrm{rpm}$ until completely dissolved. A Zinc acetate solution was prepared by dissolving zinc acetate into chitosan solution and stirred with a magnetic stirrer until homogenous. Hydrogel is made through initial Hesperidin dispersion in pectin solution while stirring. Hesperidinpectin solution, is slowly dripped into chitosan-zinc acetate mixture using $10 \mathrm{ml}$ hypodermic syringe and stirred with a magnetic stirrer (Schott model D-55122 Mainz) at $300 \mathrm{rpm}$ until hydrogel beads formed. The hydrogel is washed with $\mathrm{CO}_{2}$-free distilled water and dried at room temperature for $48 \mathrm{~h}$ [15]. Dried hydrogel undergoes evaluation, including entrapment efficiency test, power test development, in vitro drug release test, and mucoadhesive strength test. Hydrogel formula design can be seen in table 1 .

Table 1: Hesperidin hydrogel formula design

\begin{tabular}{lllll}
\hline S. No. & Composition & F1 & F2 & F3 \\
\hline 1. & Hesperidin (mg) & 50 & 50 & 50 \\
2. & Pectin (\%) & 3 & 5 & 50 \\
3. & Chitosan (\%) & 1 & 5 & 2 \\
4. & Zinc acetate (\%) & 2 & 2 & 2 \\
\hline
\end{tabular}

Pectin, chitosan, and zinc acetate solution used in every formula is $10 \mathrm{ml}$. The total volume on every formula is $30 \mathrm{ml}$. Each of the formulation is made triplicate

\section{Preparation of hesperidin standard curve}

Hesperidin standard solution is made in the concentration of 100 ppm using acetate buffer of pH 5.0, pH 6.8 buffer, $\mathrm{pH} 7.4$ buffer as the solvent (for drug test release), and in $0.3 \mathrm{M} \mathrm{NaOH}$ (for efficiency entrapment test) as presented in table 2 . Afterwards, concentration series were made in $4,10,16,22,28 \mathrm{ppm}$ for $\mathrm{pH} 5.0$ acetate buffer and $\mathrm{pH} 6.8$ phosphate buffer solvent, and concentration series of 8 , $12,16,20,24,28$ for $\mathrm{pH} 7.4$ phosphate buffer solvent and concentration series of $12,16,22,28,34,40 \mathrm{ppm}$ for $0.2 \mathrm{M} \mathrm{NaOH}$ solvent. Solution series is analyzed using UV-Vis spectrophotometer at the maximum wavelength of hesperidin. Maximum wavelength is determined using UV-Vis spectrophotometer ranging from 200 to $400 \mathrm{~nm}[16]$. The process of making standard solution concentration series is repeated for 6 times using the available standard solution. The best equation was used to calculate the drug level during in vitro drug release test [17].

\section{The entrapment efficiency test}

Dry hydrogels, which is equivalent to $50 \mathrm{mg}$ of hesperidin is placed in $0.2 \mathrm{M} \mathrm{NaOH}$ solution and settled for $24 \mathrm{~h}$. The solution is filtered using filter paper, and the filtration result is analyzed for hesperidin contents using UV-Vis spectrophotometer (Shimadzu type $2450^{\circledR}$ ) at hesperidin maximum wavelength. The result showed the amount of hesperidin entrapped inside the hydrogel matrix. The entrapment efficiency is determined by equation (1) [18].

$$
\mathrm{EE}(\%)=\frac{\text { number of obtained drug }}{\text { total drug number }} \times 100 \% \ldots \ldots
$$

\section{Swelling index}

Swelling index (SI) is performed by preparing hydrogel from each formulation, in buffer solution of $\mathrm{pH} 5.0, \mathrm{pH}$ 6.8, and $\mathrm{pH}$ 7.4. Each hydrogel is weighed at $\pm 5.0 \mathrm{mg}$, and placed in to the buffer solution. Sample buffer solution is removed at a set time interval and wet hydrogel mass was weighed. The Hydrogel swelling index is determined based on the equation below (2) [19-20].

$$
\text { SI }(\%)=\left(\frac{W s-W d}{W d}\right) \times 100 \% \ldots .
$$

For:

Ws: Swelling Hydrogel Weight

Wd: Dried Hydrogel Weight

SI: Swelling Index

\section{In vitro drug release and determination of drug release mechanism}

The in vitro hesperidin drug release from hydrogel uses USP apparatus 1 as a testing method. Dissolution medium is made using and a medium containing $2 \%$ of rat caecum. $900 \mathrm{ml}$ of the medium is used for $\mathrm{pH} 5.0$ buffer, and $\mathrm{pH} 6.8$ buffer; $100 \mathrm{ml}$ for medium containing $2 \%$ of rat caecum. In vitro drug release test was performed at $37 \pm 0,5{ }^{\circ} \mathrm{C}$ with stirring speed of $100 \mathrm{rpm}$. Drug release time in pH 5.0 buffer medium was observed for $4 \mathrm{~h}$, pH 6.8 buffer for $5 \mathrm{~h}$, and the medium contained $2 \%$ of rat caecum for $5 \mathrm{~h}$. Sampling were done on $\min 15,30,45,60 ; 90,120,180,240$ and 300, each sample is $3 \mathrm{ml}$ in volume. The taken solution is immediately replaced by a certain amount of solution from the same medium at certain time interval. The absorbance of the sample is measured using UVVis spectrophotometer at a wavelength of $283,4 \mathrm{~nm}$. In drug release test, drug release kinetic was set into zero, one, and Higuchi order, also into Korsmeyer-Peppas equation to observe the drug release mechanism. Korsmeyer-Peppas equation is shown below. (3)[21].

$$
\log \% R=\log K+n \log t \text {. (3) }
$$

For:

\section{R: released drug amounts in every $t$ \\ $K$ : constant release rate \\ $n$ : time power (showing the drug release mechanism) \\ Mucoadhesive strength}

The hydrogel mucoadhesive strength assay is based on physical equilibrium. Equipment used in this procedure, including equal-arm balance in which a beaker containing $\mathrm{pH} 7$ buffer solution is placed under the left balance disc. Fresh cow colon mucosa is used as a membrane and is attached to the mass using thread. The weight is then placed in a large beaker containing $\mathrm{pH} 7.4$ buffer solution until the solution reached the upper surface of the mucosa. Hydrogel is attached at the bottom of the left balance disc and then the disc is slowly lowered until it made a contact with cow colon mucosa. A plastic container was placed in the right balance disc and water is added using a burette with a drop rate of 100 drops/minutes. Water addition is stopped when hydrogel separates from the cow colon mucosa. The mass of water that is required to release hydrogel from cow colon mucosa was calculated as the mucoadhesive strength in grams. Equation (4) and (5) is used to calculate the hydrogen mucoadhesive strength [22]. 


$$
\begin{aligned}
& \text { Adhesion Ability }(\mathrm{N})=\frac{\text { Mucoadhesive Strength }(\mathrm{g})}{1000} \times 000 \mathrm{a} \ldots \ldots \ldots \ldots . . . .(4) \\
& \text { Mucoadhesive Strength(N/cm2) }=\frac{\text { Adhesion Ability (N) }}{\text { surface area (cm2) }} \ldots \ldots \ldots \ldots . . . . .(5)
\end{aligned}
$$

\section{RESULTS AND DISCUSSION}

Measurements using UV-Vis spectrophotometer produced a maximum wavelength of $283,4 \mathrm{~nm}$ for hesperidin. Maximum wavelength data was used to create a standard curve in various mediums and concentration to obtain an equation,which will be used to measure the number of drug releases per unit after a certain time in the drug release test.

Based on the obtained results, absorbance in $\mathrm{pH} 5.0, \mathrm{pH} 6.8, \mathrm{pH} 7.4$, and $0.2 \mathrm{MNaOH}$ medium shows good linearity with $\mathrm{r}$ (linearity) value $\geq 0,99$, where $r$ value for equation $8,9,10$, and 11 were 0.9999 , $0.9997,0.9996$, and 0.9990 respectively. The result of accuracy measurement shows the recovery value within $98.64-100.8 \%$ for $\mathrm{pH}$ 5.0 and $98.11-104.8 \%$ for $\mathrm{pH} 6.8$, within $98.75-101.47 \%$ for $\mathrm{pH} 7.4$ and $96.53-105.43 \%$ for $0.2 \mathrm{M} \mathrm{NaOH}$ medium. This result proves that every concentration is in the range of $95 \%-105 \%$. Precision measurement result shows RSD value which in the range of 0.481 $1.937 \%$ for $\mathrm{pH} 5.0$ and $0.684-1.788 \%$ for $\mathrm{pH} 6.8$, also within $0.481-$ $1.937 \%$ for $\mathrm{pH} 7.4$ and $0.551-1.290 \%$ for $0.2 \mathrm{M} \mathrm{NaOH}$ medium. These results proved that the methods used in this research are valid because the precision value did not exceed $2 \%$ [23].

\section{Entrapment efficiency (EE)}

Results showed that an increase in pectin concentration resulted in increased entrapment efficiency (EE), where formula 3 and 4 were known to have higher EE, while formula 1 has the lowest EE. Pectin has a rapid gel-forming ability and high viscosity that led to stronger hydrogel matrix and produced optimum entrapment [24-27]. Comparison of the formula is shown in fig. 2 .

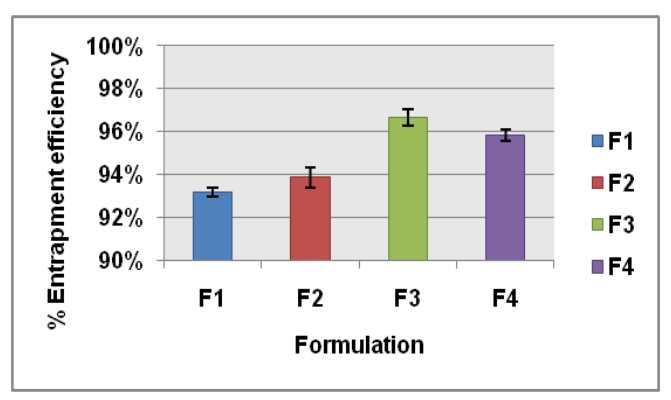

Fig. 2: Result of entrapment efficiency in $0.2 \mathrm{M} \mathrm{NaOH}$. (n= 3; mean value \pm SD)

The results shows that the analyzed data were distributed normally. This means that the standard deviation of the actual response value which separates the EE with value prediction is insignificant. EE response data showed a normal conformity model against assumptions of ANOVA. The lowest EE was $92.95 \%$ and the highest EE was $96.99 \%$. Factorial equations for the response shown in equation $6 \mathrm{EE}$.

$$
\mathrm{EP}=90,17+0,75 \mathrm{~A}-0,23 \mathrm{~B}+0,31 \mathrm{AB} \ldots \ldots(6)
$$

\section{NB: A=Variation of Pectin}

$\mathrm{B}=$ variation of chitosan

Based on equation 12, the coefficients of $\mathrm{A}$ and $\mathrm{AB}$ is positive, which means that $\mathrm{EE}$ will rise along with increasing concentration of pectin and interaction between the two polymers pectinChitosan.

\section{Swelling index}

The swelling index test aims to determine the swelling time associated with the hydrogel ability to regulate drug release within its polymer matrix. The test result shows the characteristic of hydrogel swell in pH 5.0, 6.8, and 7.4 buffer medium, the duration of swelling is positively related to hydrogel swelling ability, longer swelling time will increase the swelling percentage.

\section{Hesperidin hydrogel swelling index}

The test result showed that in $\mathrm{pH} 5.0$ hydrogel swells slowly compared to pH 6.8 and 7.4 medium (fig. 3), it can be seen in fig. 3 that formula 2 has the highest swelling percentage in $\mathrm{pH} 5.0$ medium, where chitosan concentration that is used in both formulas (formula 2 and formula 4) were higher (2\%). It is shown that in $\mathrm{pH}$ 5.0 chitosan concentration affected swelling percentages. Chitosan with pKa ranging from 6.3-6.5 is easily soluble at low $\mathrm{pH}$, where generally at low $\mathrm{pH}$ protonation of primer amine group $\left(\mathrm{NH}_{3}{ }^{+}\right)$in chitosan will occur, while pectin is unionized, so repelling force takes place inside the chitosan structure which causes significant swelling in formula with higher chitosan concentration (formula 2 and 4)[2].

Hydrogel swelling percentage at $\mathrm{pH} 6.8$ is higher than swelling percentage at $\mathrm{pH} 5.9$ acetate buffer for every formula, with formula 3 having the highest percentage of swelling, while formula 2 has the lowest swelling percentage (fig. 3). Pectin, with its characteristic as a weak acid (pKa 3-4) with the carboxylic group (-COO-) tends to ionize at higher $\mathrm{pH}$. This causes a repelling force among the carboxylate group in pectin leading to the increase of hydrogel swelling power [11]. The highest swelling percentage was achieved in pH 6.8 medium after $5 \mathrm{~h}$, at the $6^{\text {th }}$ hour, swelling percentage begin to decrease until $8^{\text {th }}$ hour. Fig. 3 also shows a higher percentage of swelling, which increases in formula with higher pectin concentration, where the highest swelling percentage was achieved by formula 3 and the lowest was formula 2 .

The higher percentage of swelling than those at $\mathrm{pH} 5.0$ and 6.8 was achieved by the sample in $\mathrm{pH} 7.4$ medium, increase in swelling percentage along with swelling time is observed, where the maximum value is recorded at the $5 \mathrm{~h}$, shown in fig. 5 . Fig. 5 shows the highest swelling percentage in the formula with higher pectin concentration, which is formula 3 and 4 , and the lowest from formula 2. The degraded hydrogel swelling percentage (after 6 hours) in pH 6.8 and 7.4 medium is related to the erosion in the hydrogel matrix. Fig. 3 shows that swelling percentage on F3 was higher in $\mathrm{pH} 6.8$ and 7.4 phosphate buffer compared to other formulas. Swelling percentage reached its maximum on the $5^{\text {th }}$ hour of the test.

This could be caused by erosion that occurred when significant swelling of hydrogel matrix loosens the matrix tissue, and after prolonged time pores forms on the matrix surface, causes in more solution entering the hydrogel. When maximum swelling occurs, there is the possibility of the drug leaking out while the matrix erodes slowly. Therefore, at the $6^{\text {th }}, 7^{\text {th }}$, and $8^{\text {th }}$ hour the swelling percentage in pH 6.8 and 7.4 medium decreases $[11,28]$. The result shows that the distribution of $\mathrm{pH} 5.0$ SI data that is analyzed is distributed normally. This means that the standard deviation of the actual response value that separates SI pH 5.0 with value prediction is insignificant. pH 5.0 SI response data distributed normally, which shows the conformity of the model against assumptions of ANOVA on the response of pH 5.0 SI. pH 5.0 model lowest SI was 34.804 and the highest was 50.011. Factorial equations for $\mathrm{pH} 5.0 \mathrm{SI}$ response is shown in equation 7 .

IP pH5,0=23,91-0,32A+14,74B-0,42AB.

NB: A = Variation of Pectin

\section{$\mathrm{B}=$ variation chitosan}

Based on equations 13,coefficient $\mathrm{A}$ and $\mathrm{AB}$ is negative, which means that the $\mathrm{pH} 5.0$ SI will decreases along with the increased concentration of the polymer, pectin, and the increasing interaction between the Chitosan and pectin polymer. Coefficient $B$ has positive value, which means that $\mathrm{pH} 5.0$ SI will rise by increasing the concentration of Chitosan. At $\mathrm{pH}$ 5.0, protonation of primary amine cluster $\left(-\mathrm{NH}_{3}{ }^{+}\right)$would occur on Chitosan, while pectin will not be ionized, so that the occurrence in the framework of Chitosan repelling led to significant development in a formula with high concentration of Chitosan (formula 2 and 4) [29]. 

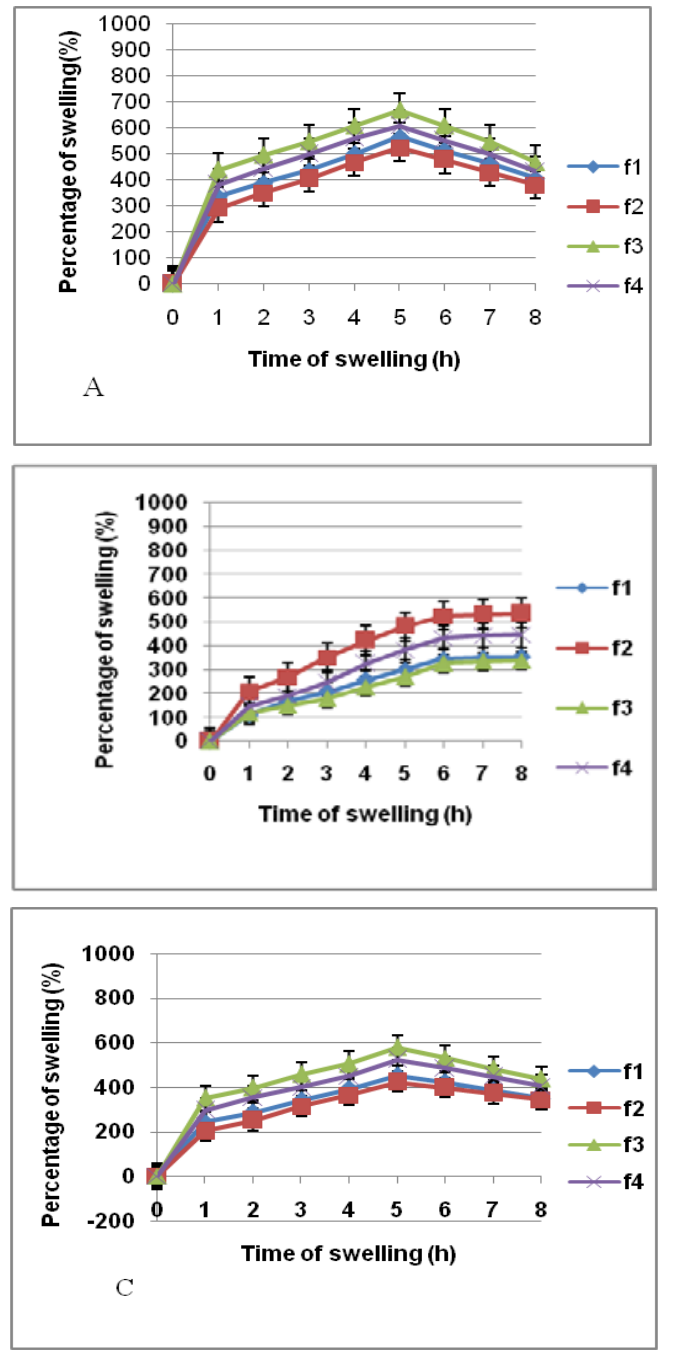

Fig. 3. Result of swelling Index in different buffer pH. Note: A: Acetic buffer (pH 5.0); B: Phosphate buffer (pH 6.8); C: Phosphate buffer (pH 7.4). ( $n=3$; mean Value \pm SD)

\section{In vitro drug release}

In vitro drug release was performed in three conditions, $\mathrm{pH} 5.0$ represents gastric condition after a meal, $\mathrm{pH} 6.8$ represents gut condition after a meal, and medium containing caecum represents colon condition.

Drug release in acetic buffer $\mathrm{pH}$ 5.0, phosphate buffer $\mathrm{pH}$ 6.8, and medium contained $2 \%$ rat

The test in $\mathrm{pH} 5.0$ medium indicates the drug release was less than $2 \%$ in every formula during a4-hour testing, even within the first hour, only $\leq 1 \%$ drug release for every formula was attained. The highest release occurs in formula 2 with the release rate reaching $1.814 \%$ in a 4 -hour test.

Lower drug release at low $\mathrm{pH}$ was consistent with studies conducted by previous researchers which concludes that pectin-chitosan combination could decrease drug release in acidic conditions [1012]. Drug release in pH 5.0 medium is shown in fig. 4.

Testing in medium $\mathrm{pH} 6.8$ shown different release rates. The highest release was achieved by formula 3 with $20.11 \%$ of release, while the lowest release is formula 1 , with $8.37 \%$ for $5 \mathrm{~h}$ of testing.

The drug release percentage was quite high in $\mathrm{pH} 6.8$ medium, possibly because, besides swelling, after $6 \mathrm{~h}$ the hydrogel also eroded [11,28], as indicated by the previous test result which is a decrease in swelling percentage after entering the $6^{\text {th }}$ hour. Drug release at pH 6.8 medium is shown in fig. 4.
Testing on medium containing $2 \%$ of rat caecum shows increased drug release was compared to two other mediums, where the percentage of drug release is between $27-56 \%$ in $5 \mathrm{~h}$ testing. Based on fig. 4 , it is known that the highest percentage of drug release was achieved by formula 3 and 4 which were $53 \%$ and $56 \%$ respectively after $5 \mathrm{~h}$ testing. Formula 3 and 4 were the formula with largest pectin concentration.

The increased percentage of drug release in the medium containing rat caecum in comparison to two other mediums were consistent with the previous study which has been done by previous researchers [30-31]. The study showed that pectinolytic or pectinase enzymes in medium containing rat caecum degrades pectin in the hydrogel matrix and broke the polymer chain, causing more pores to form on the matrix surface, making the hydrogel matrix more permeable for hesperidin $[11,22]$.

In fig 4 , drug release in medium containing $2 \%$ of rat caecum tends to be constant. This is because of hydration and pectin swelling produces viscous layer in gel layer, which results in slower drug release [32].
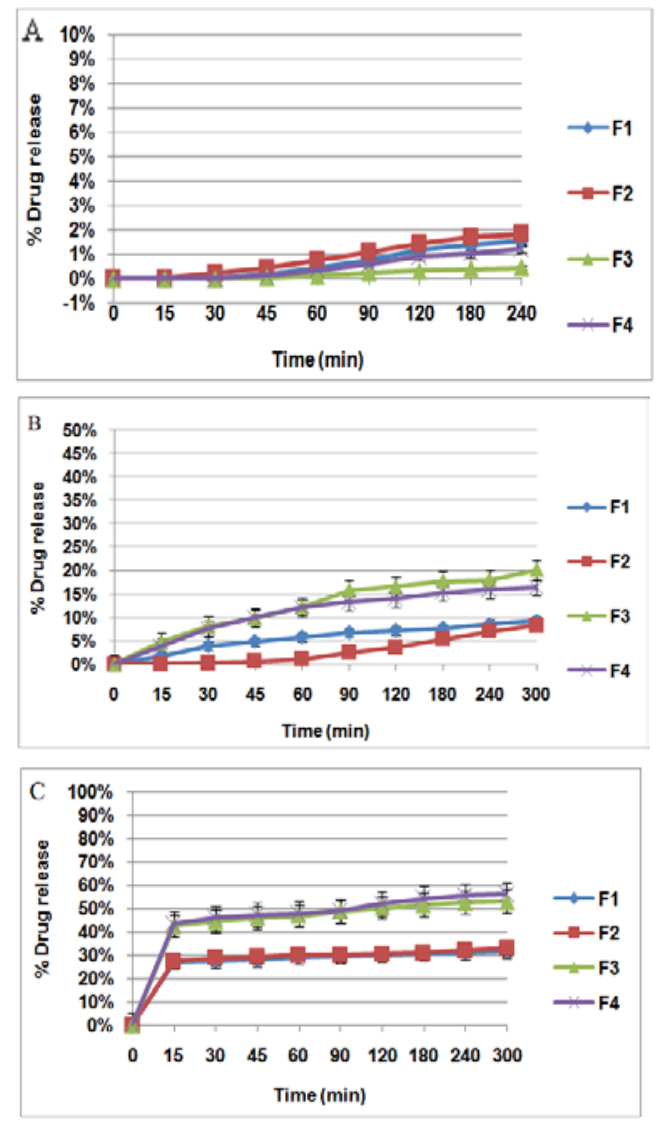

Fig. 4: Drug release on various $\mathrm{pH}$. A: Drug release in acetic buffer (pH 5.0); B: Drug release in Phosphate buffer (pH 6.8); C: Drug Release in Medium Containing $2 \%$ of Rat Caecum. $\left(\mathrm{n}_{\mathrm{a}}=3\right.$, mean value \pm SD)

The result of the drug release test is then used to observe the drug release kinetic profile against time in $\mathrm{pH} 5.0, \mathrm{pH} 6.8$ medium, and medium containing $2 \%$ of rat caecum. The result of drug release parameters can be seen in table 2 .

Measurement result shows that hesperidin release from the hydrogel matrix in medium $\mathrm{pH} 5.0, \mathrm{pH} 6.8$ and medium containing $2 \%$ of rat caecum is controlled by Higuchi kinetic release. This is based on the value of $\mathrm{r}^{2}$ in table 2 which shows that formula 1 to 4 releases the drug based on Higuchi kinetic in each medium. Higuchi kinetic describes that drug release is affected by drug diffusion through matrix pores. The drug release is shown at table 3. 
Drug release exponent (n) in Korsmeyer-Peppas equation describes the drug release mechanism which happens to the preparation of test medium. Hesperidin hydrogel with pectinchitosan polymer combination yield a n value around $1.30-1.60$ in the $\mathrm{pH} 5.0$ medium, the values were categorized as $\mathrm{n}>1$ (transport super case 2), and it showed that drug release mechanism in the preparation is controlled by relaxation ability or matrix swelling. On the contrary, hesperidin hydrogel with pectin-chitosan polymer combination gave $\mathrm{n}$ value in the range of $0.43-1.43 \mathrm{in} \mathrm{pH}$ 6.8 medium, which describes the drug release mechanism in the preparation was a combined mechanism of Fick diffusion and transport super case 2 .

Table 2: Hesperidin drug release parameter from hydrogel $\left(n_{a}=3\right.$, mean value $\left.\pm S D\right)$

\begin{tabular}{|c|c|c|c|c|c|c|}
\hline \multicolumn{7}{|c|}{ Acetic buffer pH 5} \\
\hline \multirow[t]{2}{*}{ Formula } & \multicolumn{2}{|l|}{ Zero Order } & \multicolumn{2}{|l|}{ First Order } & \multicolumn{2}{|l|}{ Higuchi } \\
\hline & $\mathbf{k}\left(\mathrm{min}^{-1}\right)$ & $\mathbf{r}^{2}$ & $\mathbf{k}\left(\min ^{-1}\right)$ & $\mathbf{r}^{2}$ & $\mathbf{k}\left(\min ^{-1}\right)$ & $\mathbf{r}^{2}$ \\
\hline F1 & $0.007 \pm 0.00$ & $0.885 \pm 0.00$ & $0.010 \pm 0.00$ & $0.700 \pm 0.09$ & $0.161 \pm 0.01$ & $0.940 \pm 0.01$ \\
\hline F2 & $0.008 \pm 0.00$ & $0.881 \pm 0.03$ & $0.009 \pm 0.00$ & $0.709 \pm 0.06$ & $0.167 \pm 0.02$ & $0.949 \pm 0.02$ \\
\hline F3 & $0.002 \pm 0.00$ & $0.895 \pm 0.03$ & $0.012 \pm 0.00$ & $0.634 \pm 0.12$ & $0.048 \pm 0.02$ & $0.945 \pm 0.01$ \\
\hline $\mathrm{F} 4$ & $0.005 \pm 0.00$ & $0.890 \pm 0.01$ & $0.011 \pm 0.00$ & $0.653 \pm 0.10$ & $0.121 \pm 0.00$ & $0.944 \pm 0.01$ \\
\hline \multicolumn{7}{|c|}{ Phosphate buffer pH 6.8} \\
\hline \multirow[t]{2}{*}{ Formula } & \multicolumn{2}{|l|}{ Zero Order } & \multicolumn{2}{|l|}{ First Order } & \multicolumn{2}{|l|}{ Higuchi } \\
\hline & $\mathbf{k}\left(\min ^{-1}\right)$ & $\mathbf{r}^{2}$ & $\mathbf{k}\left(\min ^{-1}\right)$ & $\mathbf{r}^{2}$ & $\mathbf{k}\left(\mathrm{min}^{-1}\right)$ & $\mathbf{r}^{2}$ \\
\hline F1 & $0.021 \pm 0.00$ & $0.768 \pm 0.06$ & $0.004 \pm 0.00$ & $0.583 \pm 0.04$ & $0.494 \pm 0.05$ & $0.876 \pm 0.03$ \\
\hline $\mathrm{F} 2$ & $0.031 \pm 0.00$ & $0.982 \pm 0.01$ & $0.011 \pm 0.00$ & $0.813 \pm 0.06$ & $0.711 \pm 0.10$ & $0.984 \pm 0.01$ \\
\hline F3 & $0.046 \pm 0.00$ & $0.771 \pm 0.02$ & $0.004 \pm 0.00$ & $0.637 \pm 0.02$ & $1.064 \pm 0.04$ & $0.889 \pm 0.01$ \\
\hline $\mathrm{F} 4$ & $0.035 \pm 0.00$ & $0.700 \pm 0.04$ & $0.035 \pm 0.00$ & $0.700 \pm 0.04$ & $0.837 \pm 0.07$ & $0.836 \pm 0.03$ \\
\hline \multicolumn{7}{|c|}{ Medium contained $2 \%$ rat caecum } \\
\hline \multirow[t]{2}{*}{ Formula } & \multicolumn{2}{|c|}{ Zero Order } & \multicolumn{2}{|l|}{ First Order } & \multicolumn{2}{|l|}{ Higuchi } \\
\hline & $\mathbf{k}\left(\min ^{-1}\right)$ & $\mathbf{r}^{2}$ & $\mathbf{k}\left(\min ^{-1}\right)$ & $\mathbf{r}^{2}$ & $\mathrm{k}\left(\mathrm{min}^{-1}\right)$ & $\mathbf{r}^{2}$ \\
\hline F1 & $0.015 \pm 0.00$ & $0.892 \pm 0.01$ & $0.001 \pm 0.00$ & $0.881 \pm 0.01$ & $0.337 \pm 0.04$ & $0.961 \pm 0.00$ \\
\hline F2 & $0.016 \pm 0.00$ & $0.892 \pm 0.01$ & $0.001 \pm 0.00$ & $0.877 \pm 0.00$ & $0.358 \pm 0.03$ & $0.944 \pm 0.02$ \\
\hline F3 & $0.035 \pm 0.00$ & $0.847 \pm 0.00$ & $0.001 \pm 0.00$ & $0.828 \pm 0.00$ & $0.789 \pm 0.00$ & $0.946 \pm 0.00$ \\
\hline $\mathrm{F} 4$ & $0.043 \pm 0.00$ & $0.910 \pm 0.01$ & $0.001 \pm 0.00$ & $0.894 \pm 0.01$ & $0.959 \pm 0.04$ & $0.974 \pm 0.00$ \\
\hline
\end{tabular}

Abbreviations: $\mathrm{n}_{\mathrm{a}}=$ amount of data, $\mathrm{SD}=$ deviation standard, $\mathrm{k}=$ drug release constant, $\mathrm{r}^{2}=$ coefficient of determination

Thus, drug release occurs through dissolution medium in the hydrogel matrix, under influence of matrix swelling. The $n$ value in the range of 0.0052-0.0087 were observed in the caecum medium, which describes drug release mechanism in the preparation is controlled by Fick diffusion; when the medium dissolution penetrated into the hydrogel matrix, the three dimension hydrogel network is relaxed, thus the hydrogel will swell until medium which enters the matrix could carry the drug out through the pores of the hydrogel matrix by diffusion [3334].

Table 3: Drug dissolution profile based on Korsmeyer-Peppas equation

\begin{tabular}{llll}
\hline Acetic buffer (pH 5) & & & $\mathrm{N}$ \\
\hline Formulation & $\mathrm{k}\left(\mathrm{min}^{-1}\right)$ & $\mathrm{r}^{2}$ & $1.306 \pm 0.23$ \\
F1 & $1.306 \pm 0.23$ & $0.866 \pm 0.07$ & $1.031 \pm 0.17$ \\
F2 & $1.030 \pm 0.17$ & $0.910 \pm 0.04$ & $1.606 \pm 0.71$ \\
F3 & $1.606 \pm 0.71$ & $0.801 \pm 0.09$ & $1.516 \pm 0.70$ \\
F4 & $1.516 \pm 0.71$ & $0.824 \pm 0.09$ & $\mathrm{~N}$ \\
Phosphate buffer (pH 6.8) & & & $1.191 \pm 0.59$ \\
Formulation & $\mathrm{k}\left(\mathrm{min}^{-1}\right)$ & $\mathrm{r}^{2}$ & $1.439 \pm 0.24$ \\
F1 & $0.483 \pm 0.02$ & $0.870 \pm 0.03$ & $0.454 \pm 0.02$ \\
F2 & $1.439 \pm 0.24$ & $0.970 \pm 0.02$ & $0.435 \pm 0.05$ \\
F3 & $0.454 \pm 0.02$ & $0.921 \pm 0.01$ & $\mathrm{~N}$ \\
F4 & $0.435 \pm 0.05$ & $0.857 \pm 0.01$ & 0.05 \\
Medium contained 2\% rat caecum & & & \\
Formulation & $\mathrm{k}\left(\mathrm{min}^{-1}\right)$ & $\mathrm{r}^{2}$ & \\
F1 & $0.053 \pm 0.00$ & $0.964 \pm 0.00$ & $0.054 \pm 0.00$ \\
F2 & $0.055 \pm 0.00$ & $0.954 \pm 0.02$ & $0.077 \pm 0.00$ \\
F3 & $0.078 \pm 0.00$ & $0.988 \pm 0.00$ & $0.087 \pm 0.00$ \\
F4 & $0.089 \pm 0.00$ & $0.976 \pm 0.00$ & \\
\hline
\end{tabular}

Abbreviations: $\mathrm{n}_{\mathrm{a}}=$ amount of data, $\mathrm{SD}=$ standard deviation, $\mathrm{k}=$ drug release constants, $\mathrm{r}^{2}=$ coefficient of determination, $\mathrm{n}=$ exponent of drug release, $\left(n_{a}=3\right.$, meanvalue $\left.\pm S D\right)$

\section{Mucoadhesive strength}

Mucoadhesive strength aims to determine the ability of hydrogel in sticking to the colon mucosa after swelling process. The result (fig. 5) shows that increased chitosan concentration led to the increased mucoadhesive strength of hydrogel, in which formula 2 and 4 with a higher level of chitosan concentrations (2\%) had the highestmucoadhesive strength.
Previous research which analyzes chitosan effect on rat colon mucosa showed that increased chitosan concentration will increase chitosan tendency to attach to mucosal tissue [34].

This ability to attachment occurs as a result of an electrostatic force between cationic chitosan with anionic mucous glycoprotein (sialic acid) and negative cell surface [35]. 


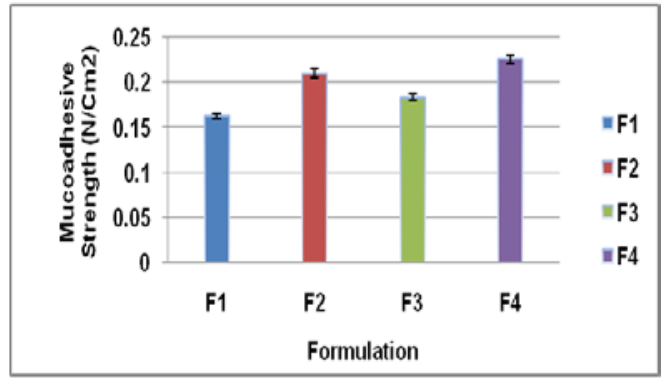

Fig. 5: Result of mucoadhesive strength test

The result shows that the distribution of the mucoadhesive strength data is distributed normally. This means that the standard deviation of the actual response value that separates mucoadhesive strength with predicted value is significant. Data of mucoadhesive strength data conforms against the assumption from ANOVA in response to mucoadhesive strength. The lowest mucoadhesive strength was $0.160 \mathrm{~N} / \mathrm{cm} 2$ and the highest was $0.230 \mathrm{~N} / \mathrm{cm}^{2}$. Factorial equations for mucoadhesive strength response is shown in equation 8.

Mucoadhesive Strength=-0.0774+0,01286A+0,0547B- $\left(2.55985 .10^{-3}\right) \mathrm{AB}$.

\section{NB: A= Variation of Pectin}

\section{$\mathrm{B}=$ variation chitosan}

Based on equation 19, it can be seen that coefficient $A$, and B has positive value, which means that mucoadhesive strength will rises, by increasing the concentration of the polymer: Chitosan and pectin. Through equations, it can be noted that the value of coefficient $B$ (Chitosan) is greater than coefficient A (pectin), this indicates that Chitosan has a greater influence in increasing the mucoadhesive strength, compared to pectin. Cationic chitosan, when interacting with mucous glycoprotein which is anionic, sustains the electrostatic force, thereby increasing the strength of the mucoadhesive [36]. The results showed the formula with the highest concentration of chitosan (formula 2 and 4) has the highest mucoadhesive strength.

\section{Data analysis result}

The response test result data was processed using Design Expert 7.0.0 trial program, with a simplex Lattice design. The program will predict the best combination from components which optimizes pectin and chitosan variations. The optimum formula that is suggested by the design was $5 \%$ pectin: $1 \%$ chitosan. Desirability values obtained for these predictions is of 0.785 which means optimum formula will yield a product with parameters or the most optimum response and liking was amounted to $78.5 \%$.

The value of the desirability of approaching 1 indicates that the actual response value will have great possibilities for significant value not unlike the response prediction results. This value is strongly influenced by the complexity of the components, the range used in the component, the number of components and response, as well as targets to be achieved in obtaining optimum formula. The image of the curve desirability can be seen in fig. 6 .

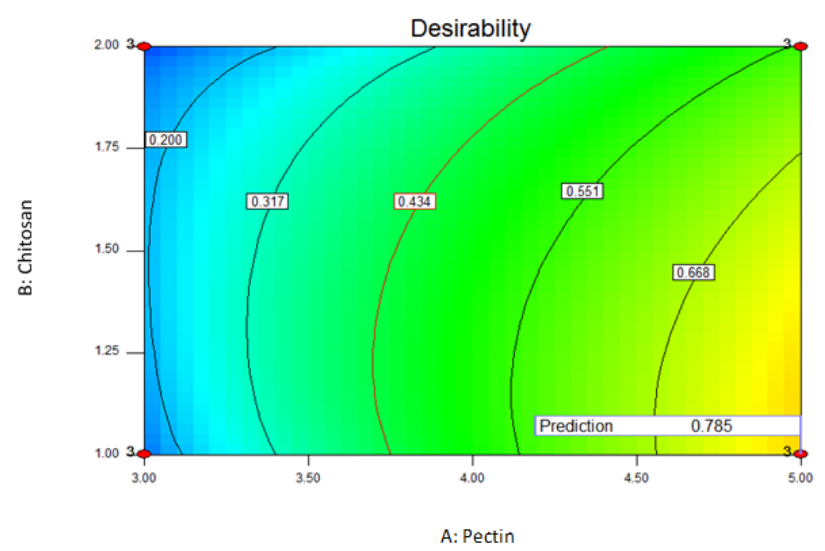

Fig. 6: Optimum hydrogel formula desirability curve

The curve above shows the tendency of desirability for the optimum formula in each comparison of pectin and chitosan. The lowest (0) and the highest value (1) were marked with the color blue to yelloworange. Based on these curves,it can be observed that the formula with a comparison of $5 \%$ pectin: $1 \%$ Chitosantends to be inside the orange-coloured area.

The lower concentration of pectin will results in the lower desirability of the formula, in which a comparison of $4 \%$ pectin: $1.5 \%$ chitosan would only yield $55 \%$ of desirability.

Table 4: Result and analysis of optimum formulation parameter

\begin{tabular}{llllll}
\hline Parameter & Prediction result & 95\%PIlow & 95\%PIhigh & Observation result & Significance \\
\hline EE & 96.656 & 95.76 & 97.55 & $96.658 \pm 0.38$ & $\mathrm{p}>0.0$ \\
SI pH5.0 & 34.949 & 33.98 & 35.92 & $34.917 \pm 0.15$ & $\mathrm{p}>0.05$ \\
SIpH6.8 & 15.773 & 15.04 & 16.49 & $15.766 \pm 0.26$ & $\mathrm{p}>0.05$ \\
SI pH 7.4 & 8.150 & 7.18 & 9.12 & $8.1460 \pm 0.32$ & $\mathrm{p}>0.05$ \\
kpH5.0 & 0.463 & 0.10 & 0.82 & $0.4610 \pm 0.14$ & $\mathrm{p}>0.05$ \\
kpH6.8 & 20.107 & 17.82 & 22.41 & $20.116 \pm 0.39$ & $\mathrm{p}>0.05$ \\
kCaecum & 52.963 & 51.26 & 54.65 & $52.955 \pm 0.63$ & $\mathrm{p}>0.05$ \\
MS & 0.183 & 0.17 & 0.19 & $0.1840 \pm 0.00$ & $\mathrm{p}>0.05$ \\
\hline
\end{tabular}

$(\mathrm{n}=3$, mean value $\pm \mathrm{SD})$ Abbreviations: $\mathrm{n}=$ number of data; =average; $\mathrm{SD}=$ standard deviation; $\mathrm{EE}=$ Entrapment Efficiency; SI= Swelling Index; $\mathrm{k}=\mathrm{drug}$ release constant; $\mathrm{PI}=$ prediction interval; $\mathrm{MS}$ = Mucoadhesive strength

Based on the result which was analyzed using independent t-test sample, it shows that actual value and prediction value from every test parameter produced by optimum formula were not significantly different due to $\mathrm{p}$-value>0.05. This indicates that the suggested equations by factorial design program method can predict the values that results in optimum hydrogel formula.

\section{CONCLUSION}

In the present study, the authors are formulating a hesperidin hydrogel using chitosan-pectin combination polymer. The study design used was a factorial design which results in 4 variations of chitosan-pectin concentration. The combination of pectin-chitosan polymer was observed in several physicochemical traits, which are entrapment efficiency, swelling index, mucoadhesive strength and drug release. Based on the observation, it is concluded that higher entrapment efficiency was achieved by formula F3 with the highest pectin concentration, which is $96.65 \%$. Highest mucoadhesive strength was achieved by formula 4 , which has the highest chitosanpectin concentration. In the mucoadhesive state, hydrogel matrix can control in vitro release of hesperidin, with the highest drug release rates achieved by the formula which have the highest pectin concentration in medium containing $2 \%$ of rat caecum, releases $56 \%$ of the drug. Hesperidin containing hydrogel release mechanism follows Higuchi's kinetics. Optimum hydrogel hesperidin formula 
was obtained by comparing pectin and chitosan concentration, which is $5 \%$ (pectin) and $1 \%$ (chitosan) respectively. Based on experimental design data, the optimum hydrogel hesperidin formula had insignificant response value between observed and predicted value ( $p$ value $>0.05$ ). Based on this study which uses hesperidin as the active substance, it can be concluded that adding a combination of chitosan-pectin could control drug releases in the target organ, which is a colon.

\section{AUTHORS CONTRIBUTION}

1. First author: Conducts formula design and physicochemical traits test.

2. The second author: Conducts drug release test and an analysis of drug release

3. The third author: Analyzes the formula and verifies the analysis method

\section{CONFLICTS OF INTERESTS}

All authors have none to declare

\section{REFERENCES}

1. Garg A, Garg S, Zaneveld LJD, Singla AK. Chemistry and pharmacology of the citrus bioflavonoid hesperidin. Phytother Res 2001;15:655-69.

2. Galati EM, Monforte MT, Kirjavainen S, Forertieri AM, Tripodo MM. Biological effects of hesperidin, a citrus flavonoid (part 1): anti-inflammatory and analgesic activity. Farmaco 1994;40:709-12.

3. Crespo ME, Galvez J, Cruz T, Ocete MA, Zarzuelo A. Antiinflammatory activity of diosmin and hesperidin in rat colitis induced by TNBS. Planta Med 1999;65:651-3.

4. Loguercio C, D'Argenio G, Delle CM. Direct evidence of oxidative damage in acute and chronic phases of experimental colitis in rats. Digest Dis Sci 1996;41:1204-11.

5. Tanaka T, Makita H, Kawabata K, Mori H, Kakumoto M, Satoh $\mathrm{K}$, et al. Chemoprevention of azoxymethane-induced rat colon carcinogenesis by the naturally occurring flavonoids, diosmin and hesperidin. Carcinogenesis 1997;18:957-65.

6. Godeberge P. Daflon-500 mg: international assessment of therapeutic interest for haemorrhoids. Drugs Today 1995;31:57-62.

7. Sansone F, Rossi A, Gaudio PD, Simone FD, Aquino RP, Lauro MR. Hesperidin gastro-resistant microparticles by spraydrying: preparation, characterization, and dissolution profiles. AAPS Pharm Sci Tech 2009;10:391-401.

8. Sadeghi M. Pectin-based biodegradable hydrogels with potential biomedical applications as drug delivery systems. J Biomater Nanobiotechnol 2011;2:36-40.

9. Liu LS, Fishman ML, Hicks KB, Kende M, Ruthel G. Pectin/zein beads for potential colon-specific drug delivery: synthesis and in vitro evaluation. Drug Delivery 2006;13:417-23.

10. Liu LS, Fishman ML, Kost J, Hicks KB. Pectin-based systems for colon-specific drug delivery via oral route. Biomaterials 2003;24:3333-43.

11. Das S, Ka-Yun Ng, Dan Paul CH. Formulation and optimization of zinc-pectinate beads for the controlled delivery of resveratrol. AAPS Pharm Sci Tech 2010;11:729-42.

12. Munjeri O, Fell JT, Collet JH. Hydrogel beads based on amidated pectins for colon-specific drug delivery: the role of chitosan in modifying drug release. J Controlled Release 1997;46:273-8.

13. Mazumder R, Allamneni Y, Firdous Sm, Parya H. Chowdhury Ad, formulation, development and in vitro release effects of ethylcellulose coated pectin microspheres for colon targeting. Asian J Pharm Clin Res 2013;6 Suppl 5:138-44.

14. Bigguci F, Luppi B, Monaco L, Cerchiara T, Zecchi V. Pectinbased microspheres for colon-specific delivery of vancomycin. J Pharm Pharmacol 2009;61:41-6.
15. Febrianisa N. Preparation and characterization of zincpectinate chitosan beads contained pentoxifylline. Undergraduate Thesis. University of Indonesia; 2012.

16. Mazzaferro LS, Breccia JD. Quantification of hesperidin in citrus-based foods using a fungal diglycosidase. Food Chem 2012;134:2338-44.

17. Hyunh-Ba K. Handbook of stability testing in pharmaceutical development: Regulation, methodologies, and best practice. Newark: Springer; 2008. p. 167.

18. Sanjay KJ. Design and development of hydrogel beads for targeted drug delivery to the colon. AAPS PharmSciTech 2007;8: E2-9.

19. Ayuningtyas F. Making and characterization of hydrogel beads from various polymers a planting medium. Undergraduate Thesis. University of Indonesia; 2012.

20. Shoushtari AM, Zargaran M. Preparation and characterization of high-efficiency ion-exchange crosslinked acrylic fibers. J Appl Polym Sci 2006;101:2202-9.

21. Vikramaditya R, Vijayavani CS, Rao VUM. Formulation and evaluation of gabapentin mucoadhesivegastroretentive tablets. Int J Pharm Anal Res 2013;2:151-62.

22. Das S, Chaudury A, Ng K. Preparation and evaluation of zincpectin-chitosan composite particles for drug delivery to the colon: the role of chitosan in modifying in vitro and in vivo drug release. Int J Pharm 2011;406:11-20.

23. LoBrutto R, Patel T. Method validation. In: kazakevich Y, LoBrutto R. Eds. HPLC for Pharmaceutical Scientists. Hoboken: Wiley Interscience; 2007.

24. Ramasamy T, Ruttala HB, Shanmugam S, Umadevi SK. Eudragitcoated aceclofenac-loaded pectin microspheres in the chronopharmacological treatment of arthritis. Drug Delivery 2013;20:65-77.

25. Dhakar VK, Chaurasia B, Kar A. Development and evaluation of floating pulsatile multi parculate drug delivery system using aceclofenac as a model drug. Int J Pharm Life Sci 2012;3:1787-96.

26. Ramteke KH, Nath L. Formulation, evaluation and optimization of pectin-bora rice beads for colon targeted drug delivery system. Adv Pharm Bull 2014;4:167-77.

27. Asha PS, Asheeba ST, Sathiskumar D. A study on microencapsulation of tamoxifen-a breast cancer drug in citrus pectin. Int J Biol Pharm Res 2015;6:19-25.

28. Chang KLB, Lin J. Swelling behaviour and the release of protein from chitosan-pectin composite particles. Carbohyd Polym 2000;43:163-9.

29. Chang KLB, Lin J. Swelling behaviour and the release of protein from chitosan-pectin composite particles. Carbohyd Polym 2000;43:163-9.

30. Sriamornsak P, Thirawong N, Weerapol Y, Nunthanid J, Sungthongjeen S. Swelling and erosion of pectin matrix tablets and their impact on drug release behaviour. Eur J Pharm Biopharm 2007;67:211-9.

31. Rubinstein A, Radai R, Ezra M, Pathak S, Rokem JS. In vitro evaluation of calcium pectinate: a potential colon-specific drug delivery carrier. Pharm Res 1993;10:258-63.

32. Viswanad V, Shammika P, Aneesh Tp. Formulation and evaluation of synthesized quinazolinone derivative for colonspecific drug delivery. Asian J Pharm Clin Res 2017;10:207-12.

33. Salyers AA, Vercellotti JR, West SEH, Wilkins TD. Fermentation of mucin and plant polysaccharides by strains of bacteroides from the human colon. Appl Environ Microbiol 1977;33:319-22.

34. Costa P, Lobo JMS. Modeling and comparison of dissolution profiles, review article. Eur J Pharm Sci 2001;13:123-33.

35. Dash S, Murthy PN, Nath L, Chowdury P. Kinetic modelling on drug release from controlled drug delivery systems. Acta Pol Pharm 2010;67:217-23.

36. Chen S, Cao Y, Ferguson LR, Shu Q, Garg S. Evaluation of mucoadhesive coatings of chitosan and thiolated chitosan for the colonic delivery of microencapsulated probiotic bacteria. J Microencapsule 2013;30:103-15.

37. Irene B, Christine V. Mucoadhesion mechanism of chitosan and thiolated chitosan-poly (isobutyl cyanoacrylate) core-shell nanoparticles. Biomaterials 2007;28:2233-43. 